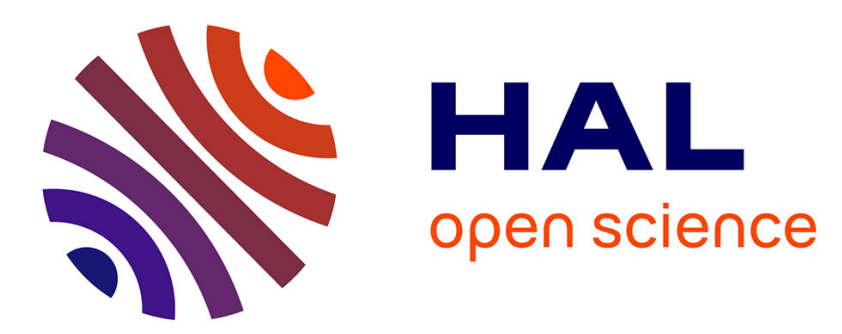

\title{
Optimisation des densités énergétiques de systèmes de stockage chimique basés sur des réactions solide-gaz renversables
}

\author{
S. Mauran, D. Bodiot, G. Crozat
}

\section{- To cite this version:}

S. Mauran, D. Bodiot, G. Crozat. Optimisation des densités énergétiques de systèmes de stockage chimique basés sur des réactions solide-gaz renversables. Revue de Physique Appliquée, 1983, 18 (2), pp.107-112. 10.1051/rphysap:01983001802010700 . jpa-00245070

\section{HAL Id: jpa-00245070 https://hal.science/jpa-00245070}

Submitted on 1 Jan 1983

HAL is a multi-disciplinary open access archive for the deposit and dissemination of scientific research documents, whether they are published or not. The documents may come from teaching and research institutions in France or abroad, or from public or private research centers.
L'archive ouverte pluridisciplinaire HAL, est destinée au dépôt et à la diffusion de documents scientifiques de niveau recherche, publiés ou non, émanant des établissements d'enseignement et de recherche français ou étrangers, des laboratoires publics ou privés. 


\title{
Optimisation des densités énergétiques de systèmes de stockage chimique basés sur des réactions solide-gaz renversables
}

\author{
S. Mauran, D. Bodiot et G. Crozat \\ Laboratoire de Chimie Minérale et de Thermochimie, Université de Perpignan, 66025 Perpignan Cedex, France
}

(Reçu le 24 mai 1982, révisé le 10 novembre, accepté le 15 novembre 1982)

\begin{abstract}
Résumé. - Le présent travail est relatif à la détermination expérimentale des capacités volumiques de stockage optimales de systèmes basés sur des réactions solide-gaz renversables.

Un modèle est proposé qui traduit l'évolution du comportement physique des composés solides au cours de la réaction. Ce modèle, vérifié expérimentalement, permet de déduire les volumes molaires de ces composés. Appliqué à divers couples chlorure-gaz (ammoniac ou méthylamine) il conduit à la détermination des densités énergétiques optimales correspondantes.
\end{abstract}

\begin{abstract}
The present work is concerned with the experimental evaluation of storage capacities for systems using reversible solid-gas reactions.

The proposed model describes the evolution, during the reaction, of the physical characteristics of the solid compounds. This model is experimentally verified and allows to determine the molar volumes of these compounds. If applied to various chloride-gas pairs, it leads to the evaluation of the best corresponding storage capabilities.
\end{abstract}

1. Introduction. - Parmi les différents systèmes de stockage de l'énergie thermique qui sont actuellement envisagés, le stockage chimique est une des voies les plus prometteuses. Le principe réside dans l'utilisation d'une ou plusieurs réactions chimiques renversables de type

$$
\sum \mathbf{P}_{i} \rightleftarrows \sum \mathbf{R}_{i}
$$

On définit le «stockage simple " par l'emploi d'une seule réaction chimique (1) même si elle s'effectue en plusieurs étapes : l'exemple le plus connu est celui de la décomposition de l'eau qui peut s'effectuer par un cycle à deux étapes utilisant la décomposition d'un oxyde :

$$
\begin{aligned}
\mathrm{MO} & \rightarrow \mathrm{M}+1 / 2 \mathrm{O}_{2} \\
\mathrm{M}+\mathrm{H}_{2} \mathrm{O} & \rightarrow \mathrm{MO}+\mathrm{H}_{2} .
\end{aligned}
$$

Dans la plupart des cas, les phases en présence sont un solide et un gaz (type décomposition d'un sulfate, hydroxyde...). Peu d'exemples utilisant des gaz seuls sont développés, à cause des problèmes de séparation; seul le système $\mathrm{SO}_{3}-\mathrm{SO}_{2}+1 / 2 \mathrm{O}_{2}$ a atteint le niveau industriel.

Dans le cas où l'un des éléments de la réaction (1) est un gaz $(G)$ on peut utiliser la chaleur intervenant dans la réaction

$$
\mathrm{G} \text { (liq.) } \rightleftarrows \mathrm{G} \text { (gaz) . }
$$

La combinaison des réactions (1) et (2) conduit, suivant les niveaux de température utilisés au déstockage, à des systèmes de stockage chimique de types "pompage thermochimique " ou "thermotransformateur » $[1,2]$.

Ces mêmes systèmes peuvent être basés sur l'utilisation de deux réactions de type (1), le gaz (G) réagissant avec deux solides de nature différente : l'efficacité des systèmes de stockage est alors améliorée.

L'ensemble de ces systèmes de stockage chimique basés sur des réactions solide-gaz connait des dévelop pements au niveau pilote de laboratoire ou industriel. Les solides employés sont des chlorures alcalinoterreux ou métalliques $[2,4,5]$ ou des sulfures [3], le gaz calorigène étant l'eau [3, 4], le méthanol [5], l'ammoniac $[2,6]$ ou la méthylamine $[2,7]$, enfin plus rarement $\mathrm{H}_{2} \mathrm{~S}$ [8].

Selon que l'on utilise seulement la réaction (1) ou que l'on fasse intervenir en plus la chaleur de condensation du gaz $\Delta H_{\mathrm{L}}(2)$, on peut définir deux densités énergétiques :

$$
D_{\mathrm{e}}^{1}=\frac{X p \Delta H}{V_{\mathrm{S}}+p V_{\mathrm{G}}} \quad \text { et } \quad D_{\mathrm{e}}^{2}=\frac{X p\left(\Delta H+\Delta H_{\mathrm{L}}\right)}{V_{\mathrm{S}}+p V_{\mathrm{G}}},
$$

où $X$ est le taux d'avancement de la réaction :

$$
\text { S. } n \mathrm{G}+p \mathrm{G} \rightarrow \mathrm{S} .(n+p) \mathrm{G}-p \Delta H,
$$


associée ou non avec :

$$
p \mathrm{G} \text { (liq.) } \rightarrow p \mathrm{G}(\mathrm{gaz})+p \Delta H_{\mathrm{L}} .
$$

$V_{\mathrm{S}}$ et $V_{\mathrm{G}}$ sont respectivement le volume occupé par une mole de composé solide, après absorption, et le volume occupé par une mole de gaz $\mathrm{G}$ à l'état liquide.

Il est proposé d'étudier ici, à partir des couples chlorure alcalino-terreux (ou métallique) et ammoniac (ou méthylamine), quelles peuvent être ces densités énergétiques suivant les conditions expérimentales imposées aux systèmes; un modèle de processus de tassement sera proposé et vérifié, le but étant d'atteindre les densités énergétiques optimales à partir d'un nombre restreint d'expériences. En effet, c'est la connaissance de ces densités énergétiques qui, liée aux critères de :

- niveau de température

- efficacité et rendement exergétique

- puissance

- coût, corrosion et nuisance

- tenue aux cyclages

autorise le choix définitif de systèmes de stockage énergétiquement les plus intéressants.

2. Dispositif expérimental. - Au cours de la réaction (3), la fixation, par le solide, du gaz G s'accompagne d'une augmentation de volume due à la dilatation du réseau cristallin. Le montage expérimental est tel qu'en empêchant cette dilatation de s'effectuer librement, on diminue la porosité du composé formé et on augmente de cette façon la densité énergétique. Pour cela on dispose des quantités connues de sel MX dans des creusets de volume donné et percés de trous permettant le passage du gaz. L'ensemble des creusets, de différents volumes, est disposé à l'intérieur d'un même réacteur. Chaque expérience consiste à envoyer le gaz $\mathrm{G}$ dans le réacteur et à suivre l'évolution de la réaction par pesée de celui-ci. Lorsque le poids du réacteur ne varie plus, on arrête l'introduction de gaz et on pèse chaque creuset. Connaissant ainsi la masse finale de composé contenue dans chaque creuset, on en déduit le taux d'avancement $X$ de la réaction pour chacun d'eux.

Si $v$ est le volume d'un creuset, on peut déterminer la densité énergétique :

$$
D_{\mathrm{e}}^{1}=\frac{X p \Delta H}{V_{\mathrm{S}}+p V_{\mathrm{G}}}=\frac{X p \Delta H}{\left(M v / m_{\mathrm{i}}\right)+p V_{\mathrm{G}}} .
$$

$m_{\mathrm{i}}$ représente la masse initiale de sel MX disposée dans le creuset et $M$ la masse molaire de MX.

$D_{\mathrm{e}}^{1}$ est une fonction du taux d'avancement $X$ et du tassement du sel $\left(m_{\mathrm{i}} / M v\right)$. Pour faciliter l'étude de cette fonction, nous avons considéré une autre expression de la densité énergétique, rapportée au seul volume du sel :

$$
D_{\mathrm{e}}^{\mathrm{s}}=X p \Delta H .\left(m_{\mathrm{i}} / M v\right) .
$$

3. Résultats et discussion. - 3.1 RÉSUlTATS EXPÉRIMENTAUX. - Les valeurs expérimentales obtenues sont représentées sous la forme de courbes $D_{\mathrm{e}}^{\mathrm{s}}=$ $f\left(m_{\mathrm{i}} / M v\right)$ qui présentent toutes la même allure de variation (Fig. 1) et notamment un maximum pour le tassement $\left(m_{\mathrm{i}} / M v\right)=\left(m_{\mathrm{i}} / M v\right)_{\max }$ caractéristique de chaque composé.

- Pour $\left(m_{\mathrm{i}} / M v\right) \leqslant\left(m_{\mathrm{i}} / M v\right)_{\max }$, on a une fonction linéaire croissante et la réaction, en fin d'expérience, est complète $(X=1)$.

$-\operatorname{Pour}\left(m_{\mathrm{i}} / M v\right)>\left(m_{\mathrm{i}} / M v\right)_{\max }, D_{\mathrm{e}}^{\mathrm{s}}$ est une fonction linéaire décroissante et le taux $X$ varie de façon continue et monotone de 1 à 0 . Au tassement $\left(m_{\mathrm{i}} / M v\right)_{\max }$ correspond la densité énergétique maximale $\left(D_{\mathrm{e}}^{\mathrm{s}}\right)_{\max }$. $\left(m_{\mathrm{i}} / M v\right)_{0}$ est la valeur du tassement au-delà de laquelle $X$ et $D_{\mathrm{e}}^{\mathrm{s}}$ sont nuls.

Il faut souligner que les résultats expérimentaux ont été obtenus en faisant varier soit $m_{\mathrm{i}} / m$, soit $v$, soit les deux simultanément. On constate que, pour un composé donné, on obtient les mêmes valeurs $D_{\mathrm{e}}^{\mathrm{s}}$ de la densité énergétique pour des valeurs identiques de $m_{\mathrm{i}} / M v$. Ceci permet de faire l'hypothèse que les résultats de cette étude, obtenus en travaillant avec des volumes de l'ordre de $75 \mathrm{~cm}^{3}$, seront applicables à des réacteurs de plus grande taille.

Nous proposons dans le paragraphe suivant un modèle permettant d'expliquer l'allure de variation des courbes $D_{\mathrm{e}}^{\mathrm{s}}=f\left(m_{\mathrm{i}} / M v\right)$.

3.2 Modèle DU PROCESSUS DE TASSEMENT. - On considère la réaction (3) dans les conditions (pression du gaz $P_{\mathrm{G}}$, température du sel $T_{\mathrm{S}}$ ) où elle est thermodynamiquement possible. Nous formulons l'hypothèse qu'à la fin de l'expérience (caractérisée par un taux d'avancement $X$ ) les composés $\mathrm{MX} . n \mathrm{G}$ et $\mathrm{MX}$. $(n+p) \mathrm{G}$ en présence atteignent respectivement les densités limites $d_{n}$ et $d_{n p}$. En effet, l'absorption de gaz par le composé MX. $n \mathrm{G}$ se traduit par le remplacement d'un réseau cristallin par un autre caractérisé par un volume de la maille plus important [9]. Ceci n'est évidemment possible que s'il reste de l'espace libre dans le creuset. Lorsque ce n'est plus le cas, les réseaux cristallins des composés solides en présence étant supposés incompressibles, la réaction s'arrête. Ces remarques nous conduisent à considérer deux possibilités :

- Dans le premier cas, le volume $v$ est suffisant pour que chaque molécule de $\mathrm{MX}$ puisse absorber successivement $n$ puis $p$ molécules de gaz G. Ceci correspond à la partie de la courbe pour laquelle $m_{\mathrm{i}} / M v \leqslant\left(m_{\mathrm{i}} / M v\right)_{\max }$. Comme la réaction est complète $(X=1)$ la courbe $D_{\mathrm{e}}^{\mathrm{s}}=f\left(m_{\mathrm{i}} / M v\right)$ est une droite de pente $p \Delta H$ (relation (4)).

- Pour un tassement supérieur $\left(m_{\mathrm{i}} / M v>\right.$ $\left.\left(m_{\mathrm{i}} / M v\right)_{\max }\right)$, le creuset est occupé, à la fin de l'expérience, à la fois par le sel $\mathrm{MX} . n \mathrm{G}$ (volume $v_{\mathrm{a}}$ ) et par le sel $\mathrm{MX} .(n+p) \mathrm{G}$ (volume $\left.v_{\mathrm{b}}\right)$. Si $M_{n}$ et $M_{n p}$ sont 


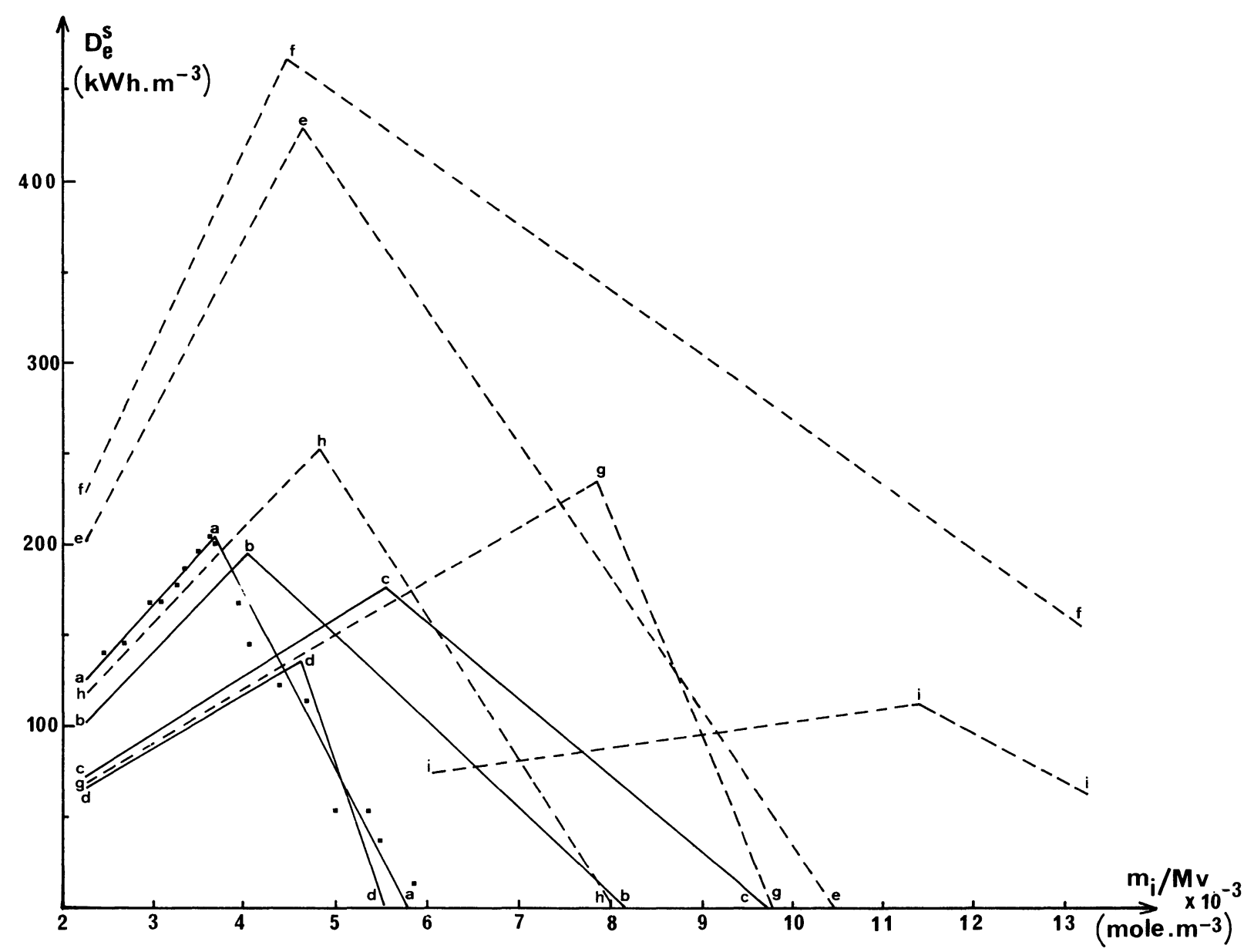

Fig. 1. - Variation de la densité énergétique en fonction du tassement du sel.

[Energy storage capacity vs. salt tamping.]

$$
\begin{array}{ll} 
& \text { a }: \mathrm{CaCl}_{2} \cdot 2 \mathrm{G}+4 \mathrm{G} \rightleftarrows \mathrm{CaCl}_{2} \cdot 6 \mathrm{G} \\
\mathrm{G}=\mathrm{CH}_{3} \mathrm{NH}_{2} & \text { b }: \mathrm{MnCl}_{2} \cdot 2 \mathrm{G}+4 \mathrm{G} \rightleftarrows \mathrm{MnCl}_{2} \cdot 6 \mathrm{G} \\
& \text { c }: \mathrm{ZnCl}_{2} \cdot 2 \mathrm{G}+2 \mathrm{G} \rightleftarrows \mathrm{ZnCl}_{2} \cdot 4 \mathrm{G} \\
& \text { d }: \mathrm{ZnCl}_{2} \cdot 4 \mathrm{G}+2 \mathrm{G} \rightleftarrows \mathrm{ZnCl}_{2} \cdot 6 \mathrm{G} \\
& \text { e }: \mathrm{BaCl}_{2}+8 \mathrm{G} \rightleftarrows \mathrm{BaCl}_{2} \cdot 8 \mathrm{G} \\
& \text { f }: \mathrm{SrCl}_{2}+8 \mathrm{G} \rightleftarrows \mathrm{SrCl}_{2} \cdot 8 \mathrm{G} \\
\mathrm{G}=\mathrm{NH}_{3} \quad & \mathrm{~g}: \mathrm{CaCl}_{2} \cdot 2 \mathrm{G}+2 \mathrm{G} \rightleftarrows \mathrm{CaCl}_{2} \cdot 4 \mathrm{G} \\
& \text { h }: \mathrm{CaCl}_{2} \cdot 4 \mathrm{G}+4 \mathrm{G} \rightleftarrows \mathrm{CaCl}_{2} \cdot 8 \mathrm{G} \\
& \text { i }: \mathrm{HgCl}_{2} \cdot 1,5 \mathrm{G}+0,5 \mathrm{G} \rightleftarrows \mathrm{HgCl}_{2} \cdot 2 \mathrm{G}
\end{array}
$$

les masses molaires respectives de ces deux sels, les densités des sels sont données par les relations :

$$
d_{n}=(1-X) \frac{m_{\mathrm{i}}}{M} \cdot \frac{M_{n}}{v_{\mathrm{a}}}
$$

et

$$
d_{n p}=X \frac{m_{\mathrm{i}}}{M} \cdot \frac{M_{n p}}{v_{\mathrm{b}}}
$$

En notant que $v=v_{\mathrm{a}}+v_{\mathrm{b}}$ on peut, à partir des relations (5) et (6) exprimer le taux d'avancement $X$ sous la forme suivante :

$$
X=\frac{d_{n} d_{n p}}{M_{n p} d_{n}-M_{n} d_{n p}} \cdot \frac{M v}{m_{\mathrm{i}}}-\frac{M_{n} d_{n p}}{M_{n p} d_{n}-M_{n} d_{n p}},
$$

$V_{n}$ et $V_{n p}$ étant respectivement les volumes molaires de MX. $n \mathrm{G}$ et de MX. $(n+p) \mathrm{G}$, on a :

$$
X=\frac{1}{V_{n p}-V_{n}} \cdot \frac{M v}{m_{\mathrm{i}}}-\frac{1}{\left(V_{n p} / V_{n}\right)-1} .
$$

Les relations (4) et (7) conduisent à l'expression suivante de la densité énergétique :

$$
D_{\mathrm{e}}^{\mathrm{s}}=\frac{p \Delta H}{1-\left(V_{n p} / V_{n}\right)} \cdot \frac{m_{\mathrm{i}}}{M v}+\frac{p \Delta H}{V_{n p}-V_{n}} .
$$

C'est l'équation d'une droite de la forme :

$$
D_{\mathrm{e}}^{\mathrm{s}}=\alpha\left(m_{\mathrm{i}} / M v\right)+\beta
$$


$\alpha$ et $\beta$ étant des constantes caractéristiques d'une réaction solide-gaz donnée. Ce résultat est conforme aux différentes expériences que nous avons effectuées.

\subsection{UTILISATION DU MODÈLE POUR LA DÉTERMINA-} TION DES COURBES $D_{\mathrm{e}}^{\mathrm{s}}=f\left(m_{\mathrm{i}} / M v\right)$. - L'application du modèle ci-dessus permet, à partir de deux points expérimentaux 1 et 2 , tels que $X<1$, de tracer l'ensemble de la courbe $D_{\mathrm{e}}^{\mathrm{s}}=f\left(m_{\mathrm{i}} / M v\right)$. Nous pouvons en effet écrire le système d'équations suivant :

$$
\left\{\begin{array}{l}
\left(D_{\mathrm{e}}^{\mathrm{s}}\right)_{1}=\frac{p \Delta H}{1-\left(V_{n p} / V_{n}\right)} \cdot\left(\frac{m_{\mathrm{i}}}{M v}\right)_{1}+\frac{p \Delta H}{V_{n p}-V_{n}}, \\
\left(D_{\mathrm{e}}^{\mathrm{s}}\right)_{2}=\frac{p \Delta H}{1-\left(V_{n p} / V_{n}\right)} \cdot\left(\frac{m_{\mathrm{i}}}{M v}\right)_{2}+\frac{p \Delta H}{V_{n p}-V_{n}} .
\end{array}\right.
$$

En le résolvant par rapport aux volumes molaires, on obtient les solutions :

$$
\begin{aligned}
V_{n}= & \frac{\left(D_{\mathrm{e}}^{\mathrm{s}}\right)_{2}-\left(D_{\mathrm{e}}^{\mathrm{s}}\right)_{1}}{\left(D_{\mathrm{e}}^{\mathrm{s}}\right)_{2} \cdot\left(\frac{m_{\mathrm{i}}}{M v}\right)_{1}-\left(D_{\mathrm{e}}^{\mathrm{s}}\right)_{1} \cdot\left(\frac{m_{\mathrm{i}}}{M v}\right)_{2}} \\
V_{n p}= & \frac{p \Delta H\left[\left(\frac{m_{\mathrm{i}}}{M v}\right)_{1}-\left(\frac{m_{\mathrm{i}}}{M v}\right)_{2}\right]+\left(D_{\mathrm{e}}^{\mathrm{s}}\right)_{2}-\left(D_{\mathrm{e}}^{\mathrm{s}}\right)_{1}}{\left(D_{\mathrm{e}}^{\mathrm{s}}\right)_{2} \cdot\left(\frac{m_{\mathrm{i}}}{M v}\right)_{1}-\left(D_{\mathrm{e}}^{\mathrm{s}}\right)_{1} \cdot\left(\frac{m_{\mathrm{i}}}{M v}\right)_{2}} .
\end{aligned}
$$

La détermination des volumes molaires $V_{n}$ et $V_{n p}$ permet ainsi de connaître les coefficients $\alpha$ et $\beta$ de la relation (9), donc de tracer la courbe $D_{\mathrm{e}}^{\mathrm{s}}=f\left(m_{\mathrm{i}} / M v\right)$.

Pour la valeur $X=0$, on trouve évidemment $D_{\mathrm{e}}^{\mathrm{s}}=0$.

D'autre part, les relations (7) et (8) conduisent, pour $X=1$, à la valeur de la densité énergétique maximale :

$$
\left(D_{\mathrm{e}}^{\mathrm{s}}\right)_{\max }=\frac{p \Delta H}{V_{n p}} .
$$

Deux aspects des résultats expérimentaux obtenus permettent de vérifier la validité du modèle proposé :

- Si on trace la courbe $D_{\mathrm{e}}^{\mathrm{s}}=f\left(m_{\mathrm{i}} / M v\right)$ à partir de deux points expérimentaux seulement (tels que $X<1$ ), l'ensemble des autres valeurs expérimentales se situe près de la courbe ainsi tracée (Fig. 1 : densité énergétique de $\mathrm{CaCl}_{2}-\mathrm{CH}_{3} \mathrm{NH}_{2}$ ).

- La comparaison (Tableau I) entre les valeurs des volumes molaires calculés d'après le modèle et celles données dans la littérature (mesurés au picnomètre en utilisant du xylène ou du kérosène [10] ou déduites des mesures de rayons $X$ [12]) montre un bon accord.

Les écarts, inférieurs en général à $10 \%$, peuvent être dûs aux incertitudes sur nos propres résultats, liées à l'expérience, en particulier dans la mesure des masses de produit avant et après réaction.

Dans le cas de $\mathrm{BaCl}_{2}$, la valeur de $V_{n}$ est exprimée à partir des résultats de Brackett et al. [12] et Vainshtein [13] qui ont déterminé deux types de structure pour $\mathrm{BaCl}_{2}$ : une structure orthorhombique et une structure cubique. La première est la plus commune (décomposition de $\mathrm{BaCl}_{2}, 2 \mathrm{H}_{2} \mathrm{O}$ à $100{ }^{\circ} \mathrm{C}$ ); la deuxième est obtenue par déshydratation de $\mathrm{BaCl}_{2}$, $2 \mathrm{H}_{2} \mathrm{O}$ à $60^{\circ} \mathrm{C}$ sous vide, et est stable jusqu'à $925-$ $960^{\circ} \mathrm{C}$ [14]. Il est remarquable de constater que, partant de $\mathrm{BaCl}_{2}$ de forme orthorhombique auquel on fait subir des réactions d'absorption et de désorption d'ammoniac, on aboutit à $\mathrm{BaCl}_{2}$ particulièrement bien cristallisé sous forme cubique $(a=7,330 \pm 0,005 \AA)$ correspondant à la maille déterminée par les auteurs $[12,13]$. La densité de $\mathrm{BaCl}_{2}$ orthorhombique est diminuée de $12 \%$ lors de la transformation en $\mathrm{BaCl}_{2}$ cubique montrant que la première réaction (solidegaz) conduit à une structure moins dense, améliorant les sites d'accueil du gaz dans la phase suivante. Cette conclusion est corroborée par un grand nombre d'expériences où nous avons constaté que la vitesse de réaction solide-gaz était bien plus importante après une absorption-désorption initiale [7].

4. Conclusion. - L'interprétation théorique de nos résultats nous permet de déterminer les volumes molaires de sels complexes (ammoniacates et aminoacates), volumes difficilement accessibles par d'autres méthodes.

L'objectif, plus pratique, de cette étude était cependant de déterminer, dans le but du stockage chimique, les densités énergétiques maximales de différents sys-

Tableau I. - Comparaison entre les volumes molaires $\left(\mathrm{cm}^{3}\right)$ calculés d'après le modèle et ceux donnés dans la littérature. Le gaz $\mathrm{G}$ est l'ammoniac.

\begin{tabular}{|l|r|r|r|r|c|}
\cline { 2 - 6 } \multicolumn{1}{c|}{} & \multicolumn{2}{c|}{ Modèle } & \multicolumn{3}{c|}{ Littérature } \\
\hline Réaction d'absorption & $V_{n}$ & $V_{n p}$ & $V_{n}$ & $V_{n p}$ & Réf. \\
\hline $\mathrm{CaCl}_{2} \cdot 2 \mathrm{G}+2 \mathrm{G} \rightarrow \mathrm{CaCl}_{2} \cdot 4 \mathrm{G}$ & 102 & 128 & 90,3 & 129,6 & {$[10]$} \\
$\mathrm{CaCl}_{2} \cdot 4 \mathrm{G}+4 \mathrm{G} \rightarrow \mathrm{CaCl}_{2} .8 \mathrm{G}$ & 125 & 207 & 129,6 & 208,2 & {$[10]$} \\
$\mathrm{HgCl}_{2} \cdot 1,5 \mathrm{G}+0,5 \mathrm{G} \rightarrow \mathrm{HgCl}_{2} \cdot 2 \mathrm{G}$ & 64 & 88 & & 80,9 & {$[11]$} \\
$\mathrm{BaCl}_{2}+8 \mathrm{G} \rightarrow \mathrm{BaCl}_{2} .8 \mathrm{G}$ & 96 & 215 & 61 & 225,8 & {$[12-13]$} \\
\hline
\end{tabular}


Tableau II. - Volumes molaires des composés étudiés, densités énergétiques maximales et volume du réservoir de liquide (pour un réacteur, contenant le solide, de $1 \mathrm{~m}^{3}$ ).

\begin{tabular}{|c|c|c|c|c|c|}
\hline Gaz & Réaction d'absorption & $\begin{array}{c}V_{n} \\
\left(\mathrm{~cm}^{3}\right)\end{array}$ & $\begin{array}{c}V_{n p} \\
\left(\mathrm{~cm}^{3}\right)\end{array}$ & $\begin{array}{c}\left(D_{\mathrm{e}}^{\mathrm{s}}\right)_{\max } \\
\mathrm{kWh} \cdot \mathrm{m}^{3}\end{array}$ & $\begin{array}{c}\text { Volume } \\
\text { du réservoir } \\
\text { de liquide } \\
\mathrm{m}^{3}\end{array}$ \\
\hline $\mathrm{CH}_{3} \mathrm{NH}_{2}$ & $\begin{array}{l}\mathrm{CaCl}_{2} \cdot 2 \mathrm{G}+4 \mathrm{G} \rightarrow \mathrm{CaCl}_{2} \cdot 6 \mathrm{G} \\
\mathrm{MnCl}_{2} \cdot 2 \mathrm{G}+4 \mathrm{G} \rightarrow \mathrm{MnCl}_{2} \cdot 6 \mathrm{G} \\
\mathrm{ZnCl}_{2} \cdot 2 \mathrm{G}+2 \mathrm{G} \rightarrow \mathrm{ZnCl}_{2} \cdot 4 \mathrm{G} \\
\mathrm{ZnCl}_{2} \cdot 4 \mathrm{G}+2 \mathrm{G} \rightarrow \mathrm{ZnCl}_{2} \cdot 6 \mathrm{G}\end{array}$ & $\begin{array}{l}172 \\
122 \\
103 \\
181\end{array}$ & $\begin{array}{l}273 \\
246 \\
180 \\
216\end{array}$ & $\begin{array}{l}204 \\
195 \\
177 \\
136\end{array}$ & $\begin{array}{l}0,64 \\
0,72 \\
0,50 \\
0,39\end{array}$ \\
\hline $\mathrm{NH}_{3}$ & $\begin{array}{l}\mathrm{BaCl}_{2}+8 \mathrm{G} \rightarrow \mathrm{BaCl}_{2} \cdot 8 \mathrm{G} \\
\mathrm{SrCl}_{2}+8 \mathrm{G} \rightarrow \mathrm{SrCl}_{2} .8 \mathrm{G} \\
\mathrm{CaCl}_{2} .2 \mathrm{G}+2 \mathrm{G} \rightarrow \mathrm{CaCl}_{2} \cdot 4 \mathrm{G} \\
\mathrm{CaCl}_{2} \cdot 4 \mathrm{G}+4 \mathrm{G} \rightarrow \mathrm{CaCl}_{2} .8 \mathrm{G} \\
\mathrm{HgCl}_{2} .1,5 \mathrm{G}+0,5 \mathrm{G} \rightarrow \mathrm{HgCl}_{2} .2 \mathrm{G}\end{array}$ & $\begin{array}{r}96 \\
57 \\
102 \\
125 \\
64\end{array}$ & $\begin{array}{r}215 \\
223 \\
128 \\
207 \\
88\end{array}$ & $\begin{array}{l}429 \\
467 \\
235 \\
253 \\
112\end{array}$ & $\begin{array}{l}0,95 \\
0,91 \\
0,40 \\
0,49 \\
0,14\end{array}$ \\
\hline
\end{tabular}

tèmes. Le tableau II résume les résultats obtenus. Ces densités énergétiques, qui varient pour les produits courants $\left(\mathrm{CaCl}_{2}, \mathrm{ZnCl}_{2}, \mathrm{BaCl}_{2}\right)$ de 130 à plus de $400 \mathrm{kWh} / \mathrm{m}^{3}$, sont à comparer à celles obtenues dans le cas d'autres systèmes de stockage de l'énergie thermique : $\left.20^{\circ} \mathrm{C}\right)$

- par chaleur sensible (eau) : $23 \mathrm{kWh} / \mathrm{m}^{3}(\Delta \theta=$

- par chaleur latente $\left(\mathrm{Na}_{2} \mathrm{SO}_{4}, 10 \mathrm{H}_{2} \mathrm{O}\right): 70 \mathrm{kWh} /$ $\mathrm{m}^{3}$ [15],

- chimique : $\mathrm{CaCl}_{2}, \mathrm{CH}_{3} \mathrm{OH}: 111 \mathrm{kWh} / \mathrm{m}^{3}$ [16], $\mathrm{Na}_{2} \mathrm{~S}, \mathrm{H}_{2} \mathrm{O}: 600 \mathrm{kWh} / \mathrm{m}^{3}$ [17],

$\mathrm{CaCl}_{2}, \mathrm{NH}_{3}$ (heptanol) : $70 \mathrm{kWh} / \mathrm{m}^{3}$

L'étude des courbes représentées à la figure 1 montre que, dans la pratique, on devra se mettre dans des conditions telles que $\left(m_{\mathrm{i}} / M v\right)<\left(m_{\mathrm{i}} / M v\right)_{\max }$, c'està-dire dans la partie croissante des courbes $D_{\mathrm{e}}^{\mathrm{s}}=$ $f\left(m_{\mathrm{i}} / M v\right)$. A densité énergétique égale, il faudra en effet une masse moins grande de réactifs que pour un tassement supérieur. Outre l'économie que cela représente sur l'achat de produits chimiques, c'est surtout au niveau de la conception des réacteurs que le compactage des produits présente un intérêt puisqu'il permet de réduire la taille, donc le coût des échangeurs.
D'autre part, si l'on se place dans l'optique du chauffage de l'habitat par pompes à chaleur chimiques [7], le volume occupé par le stockage des produits chimiques doit être compatible avec la conception d'une maison individuelle.

La densité énergétique n'étant qu'un des critères de choix du système de stockage chimique, il convient d'introduire comme critère supplémentaire la puissance du système. Nos mesures [7] montrent que la durée de la réaction totale varie de 6 à $10 \mathrm{~h}$ pour les sels non soumis à des contraintes de volume. Ces temps correspondent à des conditions expérimentales telles que les échanges thermiques entre le solide et le fluide caloporteur n'ont pas été optimisés. L'adjonction au sel d'un liant, chimiquement inerte mais à bonne conductivité thermique, permet d'augmenter les vitesses de réaction d'un facteur 2 à 3 . Ces améliorations (meilleur échangeur, addition d'un liant) seront nécessaires si l'on veut augmenter la puissance du système tout en conservant une bonne densité énergétique. Les premiers essais montrent en effet que la vitesse de réaction diminue lorsqu'augmente le compactage du sel. C'est dans cette optique que nous avons entrepris des expériences sur l'évolution des vitesses de réactions en fonction des densités énergétiques.

\section{Bibliographie}

[1] Alefeld, G., Bauer, H. C., Maier-Laxhuber, P. and ROTHMEYER, M., A zeolithe heat pump, heat transformer and heat accumulator, Int. Conf. on Energy Storage, Brighton, April 1981.

[2] Bodiot, D., Crozat, G., El Ghandour, N. and Mauran, S., Development of a Chemical Heat Pump for Solar Heating, Solar World Forum I.S.E.S., Brighton, August 1981.
[3] Dakken, K., System Tepidus, high capacity thermochemical storage/heat pump, Int. Conf. on Energy storage, Brighton, April 1981.

[4] Bourström, H. and Raldow, W., Int. J. Energy Res. 5 (1981) 43.

[5] Offenhartz, P., Brown, F. C., Mar, R. W. and Carling, R. W., J. Sol. Energy Sci. Eng. 102 (1980) 59. 
[6] Elberg, S. et Mathonnet, P., Le stockage de la chaleur sous forme d'énergie thermique à moyennes et basses températures, Colloque sur le stockage de l'énergie solaire, INSA, Lyon, janvier 1981.

[7] Mauran, S., Chauffage de l'habitat par pompes à chaleur chimiques. Détermination expérimentale des conditions thermodynamiques de fonctionnement. Thèse de 3e Cycle, Perpignan, mai 1982.

[8] Fuji, S., Kameyama, H., Yoshida, K. and KuniI, D., J. Chem. Eng. Japan 10, no 3 (1977) 224.

[9] Hartoulari, R. DE, Contribution à l'étude des systèmes solide (1) gaz-solide (2) au voisinage de l'équilibre. Thèse de Doctorat d'Etat, Dijon, 1973.

[10] Gillespie, L. J. and Gerry, H. T., J. Am. Chem. Soc. 53 (1931) 3962.

[11] Biltz, W. and Mau, C., Z. Anorg. Allg. Chem. 148 (1925) 170.
[12] Brackett, E. B., Brackett, T. E. et Sass, R. L., J. Phys. Chem. 67 (1963) 2132.

[13] Vainshtein, B. K., Dokl. Akad. Nauk SSSR 60 (1948) 1169.

[14] Gemsky, H., Neues Jahrb. Mineral. Geol. Palaeontol. 36 (1913) 513.

[15] RoYer, H., Colloque Transport et Stockage de l'Energie, Sophia-Antipolis, octobre 1978.

[16] Offenhartz, P. O'D., Int. Seminar on Thermochemical Energy Storage, Stockholm, janvier 1980.

[17] Brunberg, E. A., Int. Seminar on Thermochemical Energy Storage, Stockholm, janvier 1980.

[18] Wentworth, W. E., Int. Seminar on Thermochemical Energy Storage, Stockholm, janvier 1980. 